\section{Key to the mysteries}

\section{John M. Charap}

Einstein's Universe. By N. Calder. Pp.147. (Viking: New York; BBC Publications: London, 1979.) \$10; $£ 6.25$.

SOME of the very best popularisations of science to have been published in the past decade have had their origin in BBC television productions: these have included Nigel Calder's own Violent Universe and The Key to the Universe. And now comes, in celebration of the centenary of Albert Einstein, Einstein's Universe. As in his earlier books, $\mathrm{Mr}$ Calder has drawn upon the resources deployed by the $\mathrm{BBC}$ in their original telecast form and has made a brave effort to meet the challenge inherent in explaining the intellectual achievements of fundamental physics without either scandalising the experts, boring the lay readers for whom he writes, or still worse misleading them. That there is a very significant desire for information, a craving for a simple route to understanding, a key to the mysteries, cannot be doubted. Any physicist who has been button-holed at a cocktail party and asked to 'explain' quasars or lasers, quarks or black holes, can testify to that. But he can also give evidence of the difficulties: how to explain modern physics to an intelligent, inquisitive but non-numerate questioner whose understanding of classical physics is likely to be nonexistent. It's not just making the progression from nineteenth-century science to quantum physics and relativity, but rather going from Aristotle to Einstein leapfrogging Newton.

The millions who watched the television broadcasts may have been satisfactorily distracted by the glamour of the hardware, or entertained by Peter Ustinov's German accent, or fascinated by the contrasting personalities of the contributing scientists. And in his earlier book $\mathrm{Mr}$ Calder allowed himself some glamourous packaging, beautiful colour photographs, coffee-table format. This time his approach is more austere: indeed he seems to have taken his task to "make relativity plain" almost literally and Einstein's Universe has few illustrations, none in colour. I would even question whether the photographs chosen actually illustrate anything very significant to the purpose of the book, but am grateful for two of Einstein himself, neither of them over-familiar. Einstein thought visually, as do many creative scientists, and the book would have been better, and the author's task easier, if there had been more and better diagrams. One of the few which actually relate directly to the text (p47) is annoyingly wrong, and even if it were corrected it would still be less informative than might have been possible with better draftsmanship and captioning.

The sequence of topics chosen by $\mathrm{Mr}$ Calder to lead the reader right up to today's research is unusual and I think effective. Instead of the conventional historical approach, from special relativity to general, the order is reversed. Moving swiftly from the transverse Doppler effect to $E=m c^{2}$ and then to gravitation, the basic

\section{Sorry, for copyright reasons some images on this page may not be available online} \section{$\frac{0}{9}$ .5

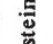

themes are blocked in. At the same time opportunity is taken whenever it is presented to make reference to the most recent experimental or observational confirmation of the consequences of the ideas. So it is that very early in the book the evidence from astronomy for the existence of black holes, as collapsed stars (Cygnus $\mathrm{X}-1$ ) or as galactic nuclei (M 87) is woven into the argument. Einstein's contributions in fields other than relativity are also brought in to further the main development of ideas. So his notion of stimulated emission leads through atomic clocks to the gravitational effect on clocks and on time. The 'time-shells', successive zones in which clocks run at greater rates as one goes further from a massive object are skilfully introduced and lead without strain to the gravitational red-shift.

The equivalence principle, so central

to Einstein's own way of thinking is ably introduced in a chapter on weightlessness: the advantage an author who can draw on the reader's experience, if only vicarious, of free-fall in a spacecapsule is very substantial and is wellexploited.

The effect of gravity on time is then used as a bridge to the effect of gravity on space. The over-worked, and to my mind misleading analogy with the distortion of a rubber sheet by heavy objects is used, but more telling is the differents in surface area of a sphere enclosing a mass from the $4 \pi r^{2}$ of Euclidean geometry-the surface of the Sun has a 'missing' area roughly the area of the USA.

The famous classic tests of general relativity, and their modern counterparts are all described; not just the perihelion advance of Mercury, but the periastron advance of the binary Pulsar $1913+16$. The book went to press when it was only possible to speculate that that same binary pulsar might give evidence through Joseph Taylor's careful and continuing observations for gravitational radiation; that confirmation was announced last December. The deflection of star-light by the Sun observed by Eddington's 1919 expedition is explained, and the counterpart annual observation of the same effect on 3C 279 also described. The so-called 'fourth classic test', the radar echo delay, is here too.

I think the treatment of the observational consequences of general relativity and the present status of such observations, is very good indeed. I'm less happy, however, with the discussion of special relativity, which is often \% made less than clear by the author's attempts to verbalise the mathematics. - However, the reasoning for the apparent rotation of a fast-moving object (and hence its foreshortening) is really very well handled, and overall I think it fair to say that many much fuller accounts of special relativity, in popular or technical treatments, succeed far less ably in getting the basic points across.

The book's last main theme is an exploration of relativistic cosmology; inevitably and quite properly this leads into more speculative areas. In the course of the book we have already had our attention directed to quantum effects, to Hawking's quantum radiance of black holes, to gravitons (there's one sentence I shall try to remember for its explanation of the non-linear effects of quantum gravity: "This incest among the gravitons produces the curvature of space"). And now we meet supergravity and gravitinos, twistors and foamy space.

I enjoyed reading the book. I was occasionally irritated by infelicities like "At its birth light accelerates infinitely 
rapidly" or "Even more fundamental than the speed of light is energy". (What exactly can that mean?). More serious a criticism is the worry that in spite of his best efforts the reader may fail to grasp the logic of the theory rather than be convinced by evidence in its favour. But Mr Calder has made a valuable contribution to the demystification of relativity, and one which is most worthy to appear in this year of Einstein's centenary.

John Charap is Professor of Theoretical Physics at Queen Mary College, University of London, $U K$.

\section{Einstein's charisma}

\section{W. B. Bonnor}

Einstein: A Centenary Volume. Edited by A. P. French. Pp. 332. (Heinemann: London; Harvard University Press: Cambridge, Massachusetts, 1979.) $£ 10.50 ; \$ 25$.

THE personality of an intellectual giant likc Einstein exerts special fascination. How far is his genius apparent in everyday behaviour? Does he have special insight into non-scientific matters? How does he get on with his ordinary fellows? It is fortunate from this point of view that Einstein's life and opinions have been so well documented: indeed so much has been written about him that it is quite hard to say anything new.

Einstein: A Centenary Volume was written at the instigation of the International Commission on Physics Education, bearing especially in mind teachers and students of physics at secondary and undergraduate levels. It consists of four parts. Pant I contains 18 reminiscences and reflections on Einstein, half of them reprinted from elsewhere. Part II is the main part of the book containing thirteen articles on Einstein's scientific work and political and social ideas. Twelve of these seem to be new, and specially commissioned for the book. Part III contains a number of letters from and to Einstein: it is not made clear which (if any) of these are being published for the first time. In Part IV a number of Einstein's writings are reprinted and a pitifully inadequate bibliography is given. The book contains many interesting photographs and drawings and is lavishly produced. However, it certainly does not offer a rounded account of Eintein's work or his personality, and the statement on the dust-cover that the book is "a total assessment of the life and works of one of the intellectual giants of our age" is unjustified. To mention one important omission, there is no description of
Einstein's work on cosmology.

In the commissioned essays there are several worthy of serious attention. Silvio Bergia gives a scholarly history of the discovery of special relativity, starting from the relativity of Newtonian physics. His article contains a careful judgement of the parts played in the discovery by Lorentz, Poincaré and Einstein, with special reference to the assessment of E. T. Whittaker, who called special relativity "the theory of Poincaré and Lorentz". Bergia concludes that Whittaker's view cannot be sustained, and that the credit usually given to Einstein is justified. There are competent accounts of Einstein and the quantum theory by Martin J. Klein, and of the observational verification of general relativity by $\mathrm{A}$. P. French.

The highlight of the book for me was the article, "Relativity theory and gravitation", by Hermann Bondi. Bondi presents a derivation of general relativity which differs from that of Einstein, and which he attributes to Fock. He starts with the notion of gravitation as a tidal force only, so that a uniform gravitational field is a zero gravitational field; and he discusses the nature of mass, describing the three different sorts and commenting on the non-existence of negative mass (of the reason for which relativity gives no clue). A somewhat

\section{Sorry, for copyright reasons some images on this page may not be available online}

sophisticated treatment of Newtonian gravitation theory is set up for later generalisation. Gravitation is introduced into special relativity by a thought-experiment using the gravitational red shift. A simple introduction to non-euclidean geometry leads painlessly to the Riemann tensor and the equation of geodesic deviation, which is neatly tied up with the Newtonian treatment and the tidal approach. It is beautifully done and should be read by all those interested in general relativity. One feels that Einstein, with his eagerness to explain relativity to the man in the street, would have enjoyed this article.

Among the essays on social and political matters are "Einstein and world affairs" by A. P. French and "Einstein and Zionism" by Gerald E. Tauber. Here Einstein emerges as the idealist, working to put the world right, but almost as much at sea as the rest of us. He espouses utopian ideas such as world government, disarmament and democratic communism; he writes to Roosevelt in 1939 calling attention to the possibility of building an atomic bomb, but later he is horrified when one is dropped; he writes to the President of the USSR Academy of Sciences asking for contributions to a book One World or None, and gets a dusty answer. All this was well meant, of course, but how naive and ineffective compared with the majesty of his achievements in physics!

In this field there emerges too a side to Einstein somewhat at variance with the saintliness which his reputation usually enjoys. Although he wrote that he passionately hated "all the loathsome nonsense that goes by the name of patriotism", yet, unable to detach himself emotionally from his Jewish origin, he became a Zionist. He went on fund-raising tours and let himself "be exhibited like a prize ox". Even before the Nazi era, Einstein disliked the Germans and after World War II he developed a pathological hatred of them, calling them mass-murderers. This attitude contrasts ill with that of his Jewish colleague Max Born who showed a gentle forgiveness for Germany after World War II.

Although, as we ought to expect, Einstein had his weaknesses, the book brings out many of his fine qualities. One is his modesty and unpretentiousness-illustrated by the story of how, at a dinner in his honour at which a too fulsome speech was being made about him, he whispered to his neighbour, referring to himself, "But he doesn't wear any socks!". 